\title{
Differential phosphoproteome of the striatum from pleiotrophin \\ knockout and midkine knockout mice treated with amphetamine: Correlations with amphetamine-induced neurotoxicity
}

\author{
Esther Gramage, Gonzalo Herradón, Yasmina B. Martín\#, Marta Vicente-Rodríguez, \\ Loreto Rojo, Heike Gnekow, Aurora Barbero, Carmen Pérez-García*
}

Pharmacology lab, Department of Pharmaceutical and Food Sciences. Facultad de Farmacia. Universidad CEU San Pablo, Madrid, Spain.

\# Current address: Departamento de Anatomía, Facultad de Medicina, Universidad Francisco de Vitoria, Pozuelo de Alarcón 28233, Spain.

\author{
*Corresponding author: \\ Carmen Pérez-García, PhD \\ Lab. Pharmacology \\ Faculty of Pharmacy \\ Universidad CEU San Pablo \\ Urb. Montepríncipe \\ 28668 Boadilla del Monte, Madrid, Spain. \\ Tel: 34-91-3724700 (Ext. 4812) \\ Fax: 34-91-3510475 \\ e-mail: capegar@ceu.es
}

\begin{abstract}
Abbreviations
Aldehyde dehydrogenase family 1 member A1 (ALDH1A1), annexin A7 (ANXA7), ATP synthase subunit alpha (ATP5A1), ATP synthase subunit d (ATP5D), COP9 signalosome subunit 5 (COPS5), creatine kinase U-type (CKMT1), delta-aminolevulinic acid dehydratase (ALAD), endoplasmic reticulum resident protein 29 (ERP29), extracellular signal-regulated kinase (ERK), fumarylacetoacetase (FAH), gamma-enolase (ENO2), GTP-binding nuclear protein Ran (RAN), immobilized metal affinity chromatography (IMAC), matrix-assisted laser desorption/ionization time-of-flight (MALDI-TOF), midkine (MK), MK-/- amphetamine (MA), MK-/- saline (MS), Parkinson's disease (PD), phosphoglycerate mutase 1 (PGAM1), pleiotrophin (PTN), PTN-/- saline (PS), PTN-/amphetamine (PA), receptor protein tyrosine phosphatase (RPTP), retinal dehydrogenase or aldehyde dehydrogenase family 1 member A1 (ALDH1A1), T-complex protein 1 subunit beta (CCT2), two-dimensional polyacrylamide gel electrophoresis (2D-PAGE), wild type (WT), WT amphetamine (WA), WT saline (WS).
\end{abstract}




\begin{abstract}
The neurotrophic factors pleiotrophin (PTN) and midkine (MK) have been shown to modulate amphetamine-induced neurotoxicity. Accordingly, PTN-/- and MK-/- mice show an increased vulnerability to amphetamine-induced neurotoxic effects. In an effort to uncover new pharmacological targets to prevent amphetamine neurotoxic effects, we have now used a proteomic approach to study protein phosphorylation, in which we combined phosphoprotein enrichment, by immobilized metal affinity chromatography (IMAC), with two-dimensional gel electrophoresis and mass spectrometry, in order to identify the phosphoproteins regulated in the striatum of PTN-/-, MK-/- and wild type (WT) mice treated with amphetamine. We identified 13 differentially expressed phosphoproteins that are judged to be relevant in the neuroprotective roles of PTN and MK against amphetamine-induced neurotoxicity. It is very interesting to note that 4 of these phosphoproteins, annexin A7 (ANXA7), COP9 signalosome subunit 5 (COPS5), aldehyde dehydrogenase family 1 member A1 (ALDH1A1) and creatine kinase U-type (CKMT1), are known to be involved in Parkinson's disease, a result of significant importance since PTN and MK have been also demonstrated to limit Parkinson's disease (PD) progress and have been suggested to be among the important genetic factors possibly preventing the development of PD in methamphetamine abusers. The data identify phosphoproteins differentially regulated by amphetamine treatment and/or the presence of endogenous PTN/MK which may be relevant mediators of PTN/MK neuroprotective effects against amphetamine-induced neurotoxicity. The data support further studies to validate the phosphoproteins here identified as possible new pharmacological targets to prevent amphetamine neurotoxic effects.
\end{abstract}

Keywords: Amphetamine; midkine; Parkinson's disease; pleiotrophin; phosphoproteins; Proteomics. 


\section{INTRODUCTION}

Amphetamine and its derivatives are drugs that are globally abused and lead to addictive behavior and severe neurological damage (Callaghan et al., 2012; Yamamoto and Bankson 2005). Hallmarks of neurotoxicity induced by these drugs include proliferation of astrocytes in striatum (Krasnova et al., 2005; Pu and Vorhees 1995), apoptosis of striatal neurons and destruction of striatal dopaminergic terminals (AresSantos et al., 2012; Granado et al., 2008a, 2008b, 2010; 2011; Krasnova et al., 2005). Increasing efforts have led to the identification of different factors that efficiently modulate the neurotoxic effects of these drugs in an effort to uncover new pharmacological targets to prevent drug-induced neurotoxicity (Ares-Santos et al., 2012; Gramage et al., 2010, 2011; Granado et al., 2011; Herradon et al., 2009). Among them, the neurotrophic factors pleiotrophin (PTN) and midkine (MK) have been shown to limit amphetamine neurotoxic effects (Gramage and Herradon, 2011). Despite the significant functional redundancy of PTN and MK (Herradon et al., 2005), amphetamine treatment in mice genetically deficient in PTN (PTN-/-) resulted in increased astrocytosis in striatum and substantia nigra, increased loss of striatal dopaminergic terminals and, surprisingly, loss of tyrosine hydroxylase-positive neurons in the substantia nigra, an effect that is not observed in wild type (WT) mice (Gramage et al., 2010). On the other hand, amphetamine-induced striatal astrocytosis is enhanced in MK-/- mice whereas loss of dopaminergic terminals in the striatum was found to be similar to that observed in WT mice (Gramage et al., 2011). These different responses to amphetamine treatment in PTN-/-, MK-/- and WT mice suggest this as an interesting model to identify druggable downstream targets in the PTN/MK signaling pathways that modulate amphetamine neurotoxic effects. 
One receptor that initiates both PTN and MK signaling pathways is the transmembrane receptor protein tyrosine phosphatase (RPTP) $\beta / \zeta$ (Meng et al., 2000; Sakaguchi et al., 2003). The interaction of PTN/MK with RPTP $\beta / \zeta$ inactivates the intrinsic tyrosine phosphatase activity of RPTP $\beta / \zeta$ leading to a rapid increase in the steady state levels of tyrosine phosphorylation of substrates of RPTP $\beta / \zeta$ (see review by Herradon and Ezquerra, 2009). Fyn kinase, one of the substrates of RPTP $\beta / \zeta$ whose levels of tyrosine phosphorylation are increased by MK/PTN, has been related to survival of dopaminergic neurons (Pariser et al., 2005a). Fyn has been shown to activate extracellular signal-regulated kinase (ERK) 1/2 signaling pathway by increasing the phosphorylation levels of ERK1/2 (Lovatt et al., 2006). Interestingly, we found a significant decrease in the phosphorylation levels of ERK1/2 in the striatum of amphetamine-treated PTN-/compared to MK-/- and WT mice (Gramage et al., 2010, 2011), suggesting diminished striatal activation of ERK1/2 could underlie the increased amphetamine-induced striatal TH loss specifically found in PTN-/- mice. Thus, the data suggest that PTN and MK neuroprotective effects could be exerted by different molecular mechanisms.

Proteomics employs high-throughput technologies that allow the investigation of proteins on a large scale in terms of their expression, function, interactions or posttranslational modifications ( $\mathrm{Li}$ and Wang, 2007). In contrast to other techniques routinely used in our laboratory, such as Western blot or immunohistochemistry, the proteomic approach does not require preselected target proteins; consequently, the results should be more objective, and could lead to the identification of novel proteins involved in the effect studied (Mann, 2008). Given the mechanism of action triggered by PTN and MK to modulate the phosphorylation levels of different proteins involved in their downstream signaling pathways, we have now used a well-established proteomic approach to study protein phosphorylation (Lee et al., 2010; Talvas et al., 2008), which includes 
immobilized metal affinity chromatography (IMAC) for phosphoprotein enrichment, twodimensional polyacrylamide gel electrophoresis (2D-PAGE) for protein separation and matrix-assisted laser desorption/ionization time-of-flight (MALDI-TOF) mass spectrometry for protein identification. In the present work, we have used this approach to perform a large scale study of protein phosphorylation in the striatum of amphetaminetreated PTN-/-, MK-/- and WT mice in an effort to dissect the different mechanisms of action triggered by PTN and MK to exert their neuroprotective roles against amphetamine-induced neurotoxicity in striatum. 


\section{METHODS AND EXPERIMENTAL PROCEDURES}

\subsection{PTN and MK genetically deficient mice}

PTN-/- and MK-/- mice were kindly provided by Dr. Thomas F. Deuel (The Scripps Research Institute, La Jolla, CA). PTN-/- mice were generated as previously described (Amet et al. 2001; Del Olmo et al., 2009). The PTN gene consists of five exons encoding an $18-\mathrm{kDa}$ protein with a 32 amino acid signal peptide. The replacement targeting vector generated a PTN null allele (PTN 2-4neo) by deleting exons 2 to 4. MK-/- mice were generated as previously described by using a basic vector to target a part of exon 1, intron 1 and a part of exon 2 of MK (Ezquerra et al., 2005, 2006; Nakamura et al., 1998). Male PTN-/-, MK-/- and WT mice on a 129/Ola x C57BL/6J background were used at 8-10 weeks of age (20-25 g). Animals were carefully distributed so the average of the animal's age was similar in every experimental group. The genotypes of PTN-/- mice were confirmed by polymerase chain reaction using as primers 5'-GAT TGA ACA AGA TGG ATT GC-3' forward and 5'-CAT TTA GGC AAA CAG GAA GGA CG-3' reverse to generate a cDNA of $\sim 0.7 \mathrm{~kb}$ detected in agarose gels from genomic DNA extracted from tails of PTN-/- and WT mice. The genotypes of the MK-/- mice were confirmed with the polymerase chain reaction using as primers 5'-ATC GGT TCC AAG TCC TCC CTC CGT C-3' forward and 5'-CAC CTT CCT CAG TTG ACA AAG ACA AGC-3' reverse to generate from genomic DNA extracted from tails of MK-/- and WT mice a cDNA of $\sim 0.7 \mathrm{~kb}$.

All the animals used in this study were maintained in accordance with European Union Laboratory Animal Care Rules (86/609/ECC directive) and the protocols were approved by the Animal Research Committee of USP-CEU.

\subsection{Amphetamine treatment}


WT, PTN-/- and MK-/- mice ( $\mathrm{n}=8$ per group) were administered with four injections of amphetamine (Sigma, Madrid, Spain) $(10 \mathrm{mg} / \mathrm{kg}$ ) or saline (control, $10 \mathrm{ml} / \mathrm{kg}$ ) with a two-hour interval between injections. This dose regimen has been previously shown to produce differential neurotoxic effects in the striatum of PTN-/-, MK-/- and WT mice (Gramage et al., 2010; 2011).

The six experimental groups depending on genotype and treatment were WT saline (WS), WT amphetamine (WA), PTN-/- saline (PS), PTN-/- amphetamine (PA), MK-/saline (MS), and MK-/- amphetamine (MA). Four days after administration of the first dose of amphetamine or saline, mice were sacrificed, since it has been previously reported that amphetamine-induced neurotoxicity in the striatum peaks 4 days after the drug administrations (Krasnova et al., 2005). Immediately after sacrifice, the mice brains removed, the striatum dissected and preserved at $-80^{\circ} \mathrm{C}$.

\subsection{Phosphoprotein identification: Proteomic analysis}

To make possible the identification of differentially phosphorylated proteins we used a proteomic approach in which we combined phosphoprotein enrichment, by immobilized metal affinity chromatography (IMAC), with two-dimensional polyacrylamide gel electrophoresis (2D-PAGE) and matrix-assisted laser desorption/ionization time-of-flight (MALDI-TOF) mass spectrometry. This approach has been previously used for nearly identical experimental purposes (Lee et al., 2010; Talvas et al., 2008). Finally, some of the differentially phosphorylated proteins detected by the proteomic techniques were tested in Western blots in individual samples.

\subsubsection{Extraction and enrichment of phosphoproteins}

For the extraction and enrichment of phosphoproteins we used the Pierce Phosphoprotein Enrichment Kit (Thermo Scientific, USA), which is based on a metal affinity chromatography (IMAC) and results in highly specific and efficient purification 
of phosphoprotein containing phosphotyrosine, phosphoserine and phosphothreonine residues (Nilsson et al. 2010). We followed the manufacturer's recommendations with slight modifications routinely used in our laboratory (Castillo et al, 2009; Sorzano et al.2008). In brief, tissue samples $(n=8)$ of each experimental group were pooled and homogenized by sonication (30 s bursts) with an ultrasonic probe (Dr. Hielscher, Germany), in $1 \mathrm{~mL}$ of the Lysis/Binding/Wash Buffer with CHAPS $(0.25 \%)$, provided by the kit, $10 \mu \mathrm{L}$ of Halt Protease Inhibitor Single-Use Cocktail EDTA-Free (Thermo Scientific, USA), and $10 \mu \mathrm{L}$ of Halt Phosphatase Inhibitor Cocktail (Thermo Scientific, USA). The samples were kept on ice to prevent over-heating during sonication. Then the samples were centrifuged $\left(10,000 \mathrm{rpm}, 20 \mathrm{~min}, 4^{\circ} \mathrm{C}\right)$ and the supernatants collected. Supernatants, containing up to $4 \mathrm{mg}$ of total protein, were then applied to a column of the kit that contained a proprietary enrichment gel and buffer, for phosphoprotein enrichment. Samples were then incubated in the column for $30 \mathrm{~min}$ at $4{ }^{\circ} \mathrm{C}$ and washed with the Lysis/Binding/Wash Buffer with CHAPS (0.25\%) to remove unbound proteins. Bound proteins were eluted with five column washes of $1 \mathrm{~mL}$ of the elution buffer provided in the kit. The pooled elution fractions were placed into the concentrator columns of the kit and centrifuged $\left(1000 \mathrm{rpm}, 4^{\circ} \mathrm{C}\right)$ for $60 \mathrm{~min}$ or until the sample volume was $150 \mu \mathrm{L}$, approximately. The concentrated phosphoprotein-enriched samples were desalted by acetone precipitation $\left(80 \% \mathrm{v} / \mathrm{v},-20^{\circ} \mathrm{C}\right.$, overnight $)$. Precipitates were finally re-suspended to a final protein concentration of $0.27 \mu \mathrm{g} / \mu \mathrm{L}$, with a buffer containing $7 \mathrm{M}$ urea, $2 \mathrm{M}$ thiourea, 4\% w/v CHAPS, $100 \mathrm{mM}$ DTT, Bio-Lyte 3/10 ampholite $0.02 \% \mathrm{v} / \mathrm{v}$, and a trace of bromophenol blue (Bio- Rad, USA). Protein concentration was measured by the Pierce BCA Protein Assay Kit (Thermo Scientific, USA).

\subsubsection{D-PAGE}


2D gel electrophoresis was carried out as described previously (Castillo et al., 2009; Zhan and Desiderio, 2003a). Briefly, $300 \mu$ of each sample obtained in the previous step were taken for the rehydration and simultaneous loading of the proteins on an IPG strip (17 cm, 3-10 NL, Bio-Rad, USA) at $50 \mathrm{~V}, 20^{\circ} \mathrm{C}$, for $12 \mathrm{~h}$ in a PROTEAN IEF cell (BioRad, USA). Then, the voltage was increased to $10000 \mathrm{~V}$ and focused for a total of 60000 Vh. Prior to SDS-PAGE, the strips were equilibrated in a solution containing $6 \mathrm{M}$ urea, 2\% SDS, $0.375 \mathrm{M}$ Tris- $\mathrm{HCl}(\mathrm{pH} 8.8$ ), and 20\% glycerol. The equilibration consisted of two steps (10 min each), DTT (2\%) was added in the first step and iodoacetamide $(2.5 \%)$ in the second one to the equilibration solution. The SDS-PAGE was run in polyacrylamide gels $(12 \%, 180 \times 200 \times 1 \mathrm{~mm})$, at $200 \mathrm{~V}, 20{ }^{\circ} \mathrm{C}$, for $6 \mathrm{~h}$ in a PROTEAN Plus Dodeca cell (Bio-Rad, USA). Then, the gels were stained with the "Silver Stain" kit (Bio-Rad, USA), according to manufacturer's protocol, which is based on the method described by Sinha et al. (2001). Silver staining was performed, in a Dodeca Stainer (BioRad, USA). By processing up to 12 gels at the same time, the Dodeca Stainer matches the capacity of the PROTEAN IEF cell and the PROTEAN Plus Dodeca cell, which streamlines the 2-D electrophoresis workflow, improves the high reproducibility of spot quality and volume provided by the PROTEAN Plus Dodeca cell (Zhan and Desiderio, 2003b) and ensures consistent results.

Gels (5 for each experimental group) were scanned using the densitometer GS-800 (Bio-Rad, USA). Spots were detected, quantified and matched automatically with the PDQuest v8 software from (Bio-Rad, USA), and manually checked. Normalization of the optical density of each spot and statistical analysis was conducted as described before (Castillo et al., 2009). Spots that showed statistical differences in optical density $(\mathrm{P}<0.05)$ between two groups, and could be well-detected visually in the gels were cut out using pipette tips for mass spectrometry identification. 


\subsubsection{Mass spectrometry analysis of protein spots}

Proteins selected for analysis were in-gel reduced, alkylated and digested with trypsin (Roche) according to Shevchenko et al. (1996). After digestion, the supernatant was collected and $1 \mu \mathrm{l}$ was spotted onto a matrix-assisted laser desorption/ionization (MALDI) sample plate and allowed to air-dry at room temperature. Then, $0.4 \mu 1$ of a 3 $\mathrm{mg} / \mathrm{ml}$ solution of alpha-cyano-4 hydroxy-cinnamic acid (Sigma, Spain) in 50\% acetonitrile, $0.1 \%$ TFA were added to the dried peptide digest spots and allowed again to air-dry at room temperature. MALDI-time of flight (TOF) mass spectrometry analyses were performed at the Proteomics Facility UCM-PCM of Madrid, a member of ProteoRed network. A 4800 Plus Analyzer MALDI-TOF/TOF mass spectrometer (Applied Biosystems, MDS Sciex, Canada) was used, operated in positive reflector mode with an accelerating voltage of $20,000 \mathrm{~V}$. All mass spectra were calibrated internally using peptides from the auto digestion of trypsin. The analysis by MALDI-TOF/TOF mass spectrometry produces peptide mass fingerprints and the peptides observed with a Signal to Noise greater than 10 can be collated and represented as a list of monoisotopic molecular weights. Proteins ambiguously identified by peptide mass fingerprints, were subjected to tandem mass spectrometry sequencing analyses. So, from the mass spectrometry spectra suitable precursor were selected for tandem mass spectrometry analyses with CID on (atmospheric gas was used) $1 \mathrm{Kv}$ ion reflector mode and precursor mass Windows +/- $5 \mathrm{Da}$. The plate model and default calibration were optimized for the tandem mass spectrometry spectra processing.

For protein identification, the non-redundant Uniprot/Swiss-Prot (release 57.15; 515203 sequences; 181334896 residues) database was searched using MASCOT 2.1 (matrixscience.com) through the Global Protein Server v3.6 from Applied Biosystems. 
Search parameters were: carbamidomethyl cystein as fixed modification and oxidized methionine as variable modification; peptide mass tolerance 50ppm for peptide mass fingerprints searched and 80-100 ppm for tandem mass spectrometry searched; 1 missed trypsin cleavage site; tandem mass spectrometry fragments tolerance $0.3 \mathrm{Da}$

We accepted positive protein identification when the probability based Mowse Score was greater than the score fixed by MASCOT as significant with $\mathrm{p}<0.05$. We also checked that theoretical MW and pI values were similar to the experimental ones.

\subsubsection{Western blot}

As described before, our proteomic approach included phosphoprotein enrichment by IMAC. This step requires employing high amounts of proteins, since typical yields after IMAC are around $10 \%$ of the total protein which, in our case, did not allow the use of individual samples and required to pool the striatum from different mice of the same experimental group. On the other hand, although IMAC produces negligible non-specific phosphoprotein purification (Nilsson et al. 2010), another concern was the possibility of detecting non-phosphorylated proteins. In addition, IMAC results in enrichment of phosphoproteins containing phosphotyrosine, phosphoserine and phosphothreonine residues (Nilsson et al. 2010) which could mask regulation of phosphorylation levels of specific residues, being of particular interest in this case phosphotyrosine residues which are highly regulated by the actions of PTN/MK on RPTP $\beta / \zeta$ (Meng et al., 2000). Therefore phosphotyrosine levels in target proteins found to be regulated in the Proteomics assays were tested in Western blots of striatum from individual samples of every experimental group.

After sacrifice, striatum from saline- and amphetamine-treated PTN-/-, MK-/- and WT mice $(n=4 /$ group), different from those used in Proteomics studies, were rapidly 
dissected, frozen in dry ice and stored to $-80^{\circ} \mathrm{C}$ until the protein extraction procedure. Tissue samples were homogenized in RIPA buffer and protein extracted in presence of protease inhibitors and a phosphatase inhibitor cocktail (Sigma, Madrid, Spain). Total protein was quantified by the Bradford protein assay (Pierce, Rockford, IL). Equilibrated protein samples were mixed with loading buffer $(60 \mathrm{mM}$ Tris $\mathrm{pH} 6.8,10 \%$ glycerol, 5\% SDS, $0.65 \% \beta$-mercaptoethanol, and $0.01 \%$ bromophenol blue), boiled for 5 minutes, and loaded onto $10 \%$ polyacrylamide gels as appropriate. The gels were transferred to nitrocellulose membranes that were blocked with $50 \mathrm{mM}$ Tris, $150 \mathrm{mM} \mathrm{NaCl}, 0.1 \%$ Tween-20 (TBS-T) and 5\% non-fat milk for 1 hour, and then probed with anti-phosphoTyr (1:1,000) antibodies (Cell signaling, Danvers, MA). Membranes were then reprobed with anti-endoplasmic reticulum resident protein 29 (ERP29) $(1: 1,000)$ antibodies (Cell signaling, Danvers, MA) or anti-gamma-enolase (ENO2) (1:1,000) antibodies (Cell signaling, Danvers, MA) to confirm the identity of the protein and to normalize the levels of tyrosine phosphorylation by the content of the target proteins in each of the individual samples. In order to normalize total protein levels of each of these proteins, membranes were re-probed with anti-actin antibodies at a 1:2,000 dilution (Chemicon, Temecula, CA). After 3 washes in TBS-T, the membranes were incubated with appropriate secondary antibodies conjugated with horseradish peroxidase diluted 1:5,000 in TBS-T with 5\% non-fat milk for $30 \mathrm{~min}$. The membranes were washed 3 times in TBS-T and the immunoreactive proteins were visualized using the ECL Enhanced method according to the manufacturer's instructions (Amersham, San Francisco, CA). Phospho-Tyr levels were quantified by densitometry in each animal sample using Image Lab image acquisition and analysis software (Bio-Rad, Hercules, CA) and normalized with total ERP29 and ENO2 protein levels. Data are presented as mean \pm standard error of the mean (S.E.M.) and were analyzed using two-way ANOVA. Relevant differences were 
analyzed pair-wise by post-hoc comparisons with Bonferroni's post-hoc tests, considering genotype and treatment as variables. $\mathrm{P}<0.05$ was considered as statistically significant. All statistical analyses were performed using graphpad prism 4 program (San Diego, CA, USA). 


\section{RESULTS}

The amphetamine regimen administrated to PTN-/-, MK-/- and WT mice has been previously demonstrated to cause different neurotoxic effects in the mouse striatum depending on the genotype (Gramage et al., 2010; 2011). Whereas amphetamine induces a significantly enhanced astrocytosis and loss of dopaminergic terminals in the striatum of PTN-/- mice compared to WT mice (Gramage et al., 2010), it only causes a significantly enhanced astrocytosis in MK-/- mice (Gramage et al., 2011). In order to correlate these genotypic differences in the vulnerability to amphetamine-induced neurotoxic effects with changes in the striatal phosphoproteome we performed proteomics studies.

For the identification of differentially phosphorylated proteins, the samples obtained after pooling 8 striatum of every experimental group (WS, WA, PS, PA, MS and MA) were first enriched in phosphoproteins by IMAC. The phosphoprotein yield ranged from 8.02 to $12.5 \%$ of the total protein content. Proteins were then separated by 2D-PAGE. The gels ( $\mathrm{n}=5$ per group) obtained from the 6 experimental groups were analyzed simultaneously and matched in the same set. The staining detection limit achieved in the silver stained gels allowed the visualization of faint spots, however for the statistical analysis we considered only well-resolved spots, with an optical density higher than 50 a.u. that could be well visualized, manually excised and, therefore, analyzed by mass spectrometry. Of all spots that showed significant differences in normalized optical density among the different experimental groups, we selected those with an expression fold-change of 1.5 or more. Finally, 14 spots, corresponding to 13 different proteins, were successfully identified by either peptide mass fingerprinting or tandem mass spectrometry (Table 1). Figure 1 displays all identified spots and their position in one representative 
gel. The phosphorylation of all these proteins in different residues has been previously demonstrated according to PhosphositePlus (Hornbeck et al., 2012; see Table 1).

When comparing the 2-D patterns of expression between different treatments within the same genotype, 5 spots showed significant differences in optical density in WA $v s$. WS, 3 spots in PA vs. PS and 7 spots in MA vs. MS (Table 2). In WT mice, 4 of these 5 proteins were significantly down-regulated after the treatment with amphetamine. In contrast, amphetamine treatment resulted in a significant upregulation of 7 out of the 10 proteins found to be significantly regulated by amphetamine treatment in both PTN-/- and MK-/- mice.

Considering genotypic differences within the same treatment (saline or amphetamine), we identified significant differences in the optical density of 6 spots in PS $v s$. WS analysis, 8 spots in MS vs. WS, 9 spots in PA $v s$. WA and 6 spots in MA vs. WA (Table 2). In addition, statistical differences were detected in 6 spots in MS vs. PS and 6 spots in MA vs. PA analysis (Table 2).

Interestingly, most of the proteins showed a significant decrease in optical density in control (saline-treated) knockout animals (PS and MS groups) when compared with WS animals (5 out of 6 in PS, and 7 out of 8 in MS). This result is consistent with the anticipated molecular effects of genetic deletion of these cytokines since PTN and MK are known to increase the phosphorylation in tyrosines through their ability to block the intrinsic phosphatase activity of their common receptor RPTP $\beta / \zeta$ (Herradon and Ezquerra, 2009). As discussed in the discussion section of this manuscript, most of the 13 proteins identified in our proteomic analysis are judged to be relevant in the neuroprotective roles of PTN and MK against amphetamine-induced neurotoxicity. However, as representative of these proteins, we chose to further study the pattern of tyrosine phosphorylation in gamma-enolase (ENO2), by performing Western blots in 
samples from 4 individual animals per experimental group. Neither the nonphosphorylated nor the tyrosine-phosphorylated form of ENO2 showed significant changes of expression between the different experimental groups (Figure 2).

In addition, we also decided to study the pattern of tyrosine phosphorylation in endoplasmic reticulum resident protein 29 (ERP29) because it was the only protein significantly over-expressed in saline and amphetamine-treated knockout mice whereas proteomics studies failed to detect readable levels of ERP29 in WT mice independently of the treatment considered. In Western blots, ERP29 showed a similar pattern of expression to that uncovered in the proteomic analysis. Tyrosine phosphorylation levels normalized by total amounts of ERP29 were found to be increased in amphetaminetreated knockout mice compared with WT mice, group in which readable levels of tyrosine phosphorylation were not detected (Figure 3). In addition, total ERP29 protein levels normalized by actin levels in each experimental group did not differ in salinetreated groups; however, ERP29 protein levels were significantly increased in amphetamine-treated PTN-/- and MK-/- mice compared with WT mice (Fig. 3). 


\section{DISCUSSION}

The neurotrophic factors PTN and MK have been shown to modulate drugs of abuseinduced neurotoxicity (Gramage and Herradon, 2011). Accordingly, genetic deletion of PTN or MK increases the vulnerability to amphetamine-induced neurotoxic effects. These neurotoxic effects of amphetamine are significantly enhanced in PTN-/- mice, animals in which an enhanced astrocytosis is accompanied by a significant loss of striatal dopaminergic terminals compared to WT mice (Gramage et al., 2010). Amphetamine also induces an increased striatal astrocytosis in MK-/- mice but its effects on dopaminergic terminals do not differ from those found in WT mice (Gramage et al., 2011). As a result, a classification of these genotypes according to their vulnerability to amphetamineinduced neurotoxic effects can be done in this animal model: PTN-/- > MK-/- > WT. Our aim was to use this model to identify new pharmacological targets to prevent amphetamine neurotoxic effects among the phosphoproteins regulated in the striatum of mice from the 3 genotypes treated with amphetamine. This strategy follows previous efforts by our group to discriminate among the known substrates of the PTN/MK receptor $\mathrm{RPTP} \beta / \zeta$ whose levels of phosphorylation are regulated by PTN and/or MK and are important in these cytokines neuroprotective effects (Gramage et al. 2010; 2011). In those studies, we tested in Western blots the phosphorylation levels of TrkA, Fyn kinase and ERK1/2 in striatum from amphetamine-treated PTN-/-, MK-/- and WT mice. However the targeted nature of this strategy limits the identification of other changes than previously anticipated (Mann, 2008). For this reason in the present work we decided to perform a large scale and non-targeted study of protein phosphorylation by employing proteomic techniques in our animal model.

In the present studies we have combined IMAC, 2D-PAGE and mass spectrometry, an approach that has been successfully used to study protein phosphorylation by others 
(Talvas et al., 2008; Lee et al., 2010). In the resulted gels, we obtained well resolved spots (Figure 1) that were compared between treatments within the same genotype and between genotypes within the same treatment. Nine comparisons were made among the 6 experimental groups that finally resulted in the successful identification of 14 spots that were differently expressed between genotypes, treatments or both (Table 2). The 14 identified spots actually corresponded to 13 different proteins; thus, spots 8504 and 9501 were the same protein, ATP synthase subunit alpha (ATP5A1); however the pattern of changes was not the same for both spots. These spots are very close in the gel, and slightly differ in their horizontal position (see Figure 1) and, therefore, in their pI. Changes in the $\mathrm{pI}$ of a protein are usually due to differences in the number or in the type of the phosphorylated residues (Gorg et al., 2004).

Before discussing in detail the potential relevance of the proteins identified, it is interesting to discuss several general conclusions. First, when the three genotypes treated with saline are compared, most of the proteins showing significant differences between knockout and WT mice were found to be down-regulated in knockout animals (Table 2) suggesting lack of PTN or MK leaves unchecked the intrinsic phosphatase activity of $\mathrm{RPTP} \beta / \zeta$ causing a general decrease in phosphorylation of proteins, especially in residues whose phosphorylation levels are known to be highly regulated by PTN/MK such as tyrosine (Meng et al., 2000) and serine (Pariser et al., 2005b). Secondly, and surprisingly, amphetamine treatment was found to cause the opposite effect; most of the phosphoproteins differentially expressed in PTN-/- and MK -/- mice, compared to WT, where up-regulated in the knockout animals treated with amphetamine. Finally, as expected given the well documented functional redundancy between PTN and MK (Herradon et al., 2005), some of the identified proteins showed a similar pattern of changes of expression in PTN -/- and MK -/- mice. However, other proteins were 
differentially affected in both genotypes which could be relevant to uncover the molecular mechanisms underlying the previously described functional differences between PTN and MK in the modulation of the neurotoxic effects induced by amphetamine (Gramage et al., 2010; 2011).

We decided to test in Western blots two of the identified proteins of special interest since the possibility of detecting non-phosphorylated proteins should not be totally discarded and, importantly, the phosphoprotein enrichment by IMAC does not discriminate between the different phosphorylated residues. ENO2 was chosen because of its well documented neuroprotective effects ( $\mathrm{Li}$ et al., 2009; Obermajer et al. 2009) together with being one of the down-regulated phosphoproteins in both PS and MS groups compared with WS. Moreover, this protein was up-regulated after amphetamine treatment only in the knockout mice (Table 2), suggesting a possible role of ENO2 in the exacerbated amphetamine-induced neurotoxic effects in striatum of knockout mice. We did not find significant changes between genotypes or treatments in the levels of tyrosine phosphorylation of ENO2 nor in the total ENO2 protein levels, suggesting the differences identified in Proteomics studies could be caused by differences in the phosphorylation levels of serine and threonine residues. All in all, previous evidence showing the neurotrophic and neuroprotective properties of ENO2 (Li et al., 2009; Obermajer et al. 2009) together with the proteomics studies presented here support further studies of the role of ENO2 in amphetamine-induced neurotoxicity. In support of this hypothesis, PTN was previously shown to limit amphetamine-induced loss of PC12 cell viability (Gramage et al. 2010b) and, in different studies, activation of ENO2 has been shown to increase PC12 cell survival (Obermajer et al. 2009), suggesting that basal deficiency in overall phosphorylation of this enzyme in the striatum of knockout mice (PS and MS groups) could play a role in the increased vulnerability of these mice to amphetamine- 
induced neurotoxic effects, being possibly amphetamine-induced upregulation of phospho-ENO2 in these knockout mice part of a compensatory mechanism.

We also tested the tyrosine phosphorylation levels of ERP29 in Western blots, since it was the only protein significantly over-expressed in knockout mice compared to WT independently of the treatment considered (Table 2). We found an interesting correlation with Proteomics studies. Tyrosine phosphorylation levels of ERP29 were found to be significantly increased in both PTN-/- and MK-/- mice treated with amphetamine whereas we failed to detect readable tyrosine phosphorylation levels of ERP29 in WT mice. Importantly, total ERP29 protein levels were perfectly readable in WT mice treated with amphetamine although in significant lower levels than those found in PTN-/- and MK-/treated with amphetamine. ERP29 is an endoplasmic reticulum derived chaperone, highly expressed throughout the brain, which is thought to have an important role in the development and preservation of neurons, as well as in plasticity and synapse formation (MacLeod et al. 2004; Tang et al., 2011). Taken together, the data suggest further consideration for ERP29 as a possible modulator of PTN/MK neuroprotective effects against amphetamine-induced neurotoxicity.

PTN and MK have been demonstrated to modulate amphetamine neurotoxic effects in the nigrostriatal pathway (Gramage et al., 2010; 2011; Gramage and Herradon, 2011), have been shown to limit Parkinson's disease (PD) progress (Taravini et al., 2011; Prediger et al., 2011) and have been suggested to be important genetic factors to be studied in the increased population of methamphetamine abusers that develop PD as a result of drug abuse (Gramage and Herradon, 2011; Callaghan et al., 2012). It is very interesting to note that several proteins known to be involved in PD can be found in the list of proteins differentially regulated in the present work. The phosphorylation of Annexin A7 (ANXA7) was differentially modulated in PTN-/- and MK-/- compared to 
WT: it decreased in PA and MS groups and was not affected in PS and MA animals (Table 2). ANXA7 is a calcium/phospholipid-binding protein involved in exocytosis (Liemann et al., 1995) whose changes of expression have been related to PD (Lessner et al., 2010). COP9 signalosome subunit 5 (COPS5), which was found to be significantly upregulated in the striatum of amphetamine-treated MK-/- mice, is known to regulate the cellular ubiquitin/proteasome pathway which is important for lysis of mal-functioning and abnormal proteins ( $\mathrm{Li}$ et al. 2008). Ubiquitin/proteasome pathway disruptions have a role in neurodegenerative diseases such as PD (Huang and Figueiredo-Pereira 2010), and are thought to be involved in neurotoxicity following methamphetamine injection ( $\mathrm{Li}$ et al. 2008). Retinal dehydrogenase or aldehyde dehydrogenase family 1 member A1 (ALDH1A1), which is down-regulated in PS and MS compared with WS and in WA and MA compared with PA, is involved in the metabolism of biogenic aldehydes and amines, such as dopamine, and its potentially toxic derivatives (Anderson et al., 2011) and its levels of expression are significantly regulated in the substantia nigra of Parkinson's disease patients (Westerlund et al., 2005) and in animal models of PD (Wey et al., 2012). Finally, the phosphorylation of creatine kinase U-type (CKMT1), the octameric mitochondrial form (also stated as mitCK) of creatine kinase (CK) in the brain, was found to be decreased only in WT mice after amphetamine treatment. CKMT1 is involved in the regulation of $\mathrm{Ca} 2+$ homeostasis and shielding effects on the opening of transition permeability pores in the mitochondria, thus affecting necrotic and apoptotic processes, suggesting alterations in mitCK levels may play a role in neurodegenerative diseases (Nersesova, 2011). The data suggest ANXA7, COPS5, ALDH1A1 and CKMT1 as potential modulators of PTN/MK actions in amphetamine-induced neurotoxicity, PD and in their connection roles between drug addiction and PD. 
Other interesting proteins detected in our study where subunit alpha (ATP5A1) and subunit d (ATP5D) of ATP synthase (Table 2). Alterations in ATP synthase have been previously described after treatment with amphetamine and related to impaired cellular energy metabolism that results in an increase in the production of reactive oxygen species which in turn causes apoptosis and necrosis in nerve cells (Brown and Yamamoto, 2003). We have already mentioned that ATP5A1 was detected in 2 spots that may correspond to 2 different positions of residue phosphorylation. Interestingly, we observed an opposed pattern of expression changes for these spots. Thus, the expression of spot 8504 was decreased in knockout mice compared to WT after saline administration, but expression of spot 9501 was up-regulated in knockout mice compared to WT after amphetamine administration. The data suggest that changes in the pattern of phosphorylation of specific tyrosine, serine and threonine residues of ATP5A1 may define the final contribution of ATP5A1 to the neuroprotective effects of PTN and MK against amphetamine-induced neurotoxicity.

T-complex protein 1 subunit beta (CCT2) is a chaperone involved in tubulin biogenesis (Spiess et al., 2004) which is upregulated in PA and MA groups compared to WA (Table 2), suggesting a possible role for this protein in the exacerbated neurotoxic effects of amphetamine in absence of endogenous PTN or MK. Phosphoglycerate mutase 1 (PGAM1) is an enzyme involved in glycolysis (Narayanan et al., 2004) and was found to be significantly down-regulated in $\mathrm{MK}-/-$ mice independently of the treatment considered. Interestingly, PGMA1 was also found to be down-regulated in a mouse model of methamphetamine-induced dopaminergic neurotoxicity (Xie et al., 2002). Delta-aminolevulinic acid dehydratase (ALAD) and fumarylacetoacetase (FAH) were found to be significantly down-regulated in PTN-/- mice independently of the treatment considered (table 2), which was especially exacerbated in the case of FAH, found to be 
undetectable in PTN-/- mice. FAH is the final enzyme in the tyrosine degradation pathway and its deficiency causes a serious disorder called type I tyrosinemia. In this disease, the toxic metabolites derived from tyrosine degradation inhibit the hemesynthesis at the level of the step catalyzed by ALAD, which in turn causes polyneuropathy (Scott, 2006). The failure to detect the phosphorylated, possibly activated, forms of these two proteins in the most vulnerable genotype (PTN-/-) to amphetamine-induced neurotoxic effects in the striatum strongly suggest an important role of FAH and ALAD in the neuroprotective actions triggered by PTN.

Finally, GTP-binding nuclear protein Ran (RAN) is a member of the Ras family of small GTPases that orchestrates multiple cellular responses (Clarke and Zhang, 2008; Cook and Conti, 2010) which is found to be upregulated in WT and PTN-/- mice after amphetamine treatment. To the best of our knowledge, RAN had not been previously associated with drugs of abuse effects.

\section{CONCLUSIONS}

We have identified phosphoproteins differentially regulated by amphetamine treatment and/or the presence of endogenous PTN/MK which may be relevant mediators of PTN/MK neuroprotective effects against amphetamine-induced neurotoxicity. The data support further studies to validate the phosphoproteins here identified as possible new pharmacological targets to prevent amphetamine neurotoxic effects. 
Acknowledgements: This work has been supported by grant SAF2009-08136 from Ministerio de Ciencia e Innovación of Spain to GH. EG is supported by fellowship AP2008-00726 from the Spanish Ministerio de Ciencia e Innovación. HG and LR are students from the University of Bath (UK) and Surrey (UK), respectively, supported by Erasmus fellowships.

\section{Conflict of Interest statement}

The authors declare that there are no conflicts of interest. 


\section{REFERENCES}

Amet, L.E., Lauri, S.E., Hienola, A., Croll, S.D., Lu, Y., Levorse, J.M., Prabhakaran, B., Taira, T., Rauvala, H., Vogt, T.F., 2001. Enhanced hippocampal long-term potentiation in mice lacking heparin-binding growth-associated molecule. Mol Cell Neurosci 17, 1014-1024.

Anderson, D. W., Schray, R. C., Duester, G., Schneider, J. S, 2011. Functional significance of aldehyde dehydrogenase ALDH1A1 to the nigrostriatal dopamine system. Brain Research. 1408, 81-87.

Ares-Santos, S., Granado, N., Oliva, I., O'Shea, E., Martin, E.D., Colado, M.I., Moratalla, R., 2012. Dopamine $\mathrm{D}(1)$ receptor deletion strongly reduces neurotoxic effects of methamphetamine. Neurobiol Dis. 45(2), 810-820.

Brown, J. M., Yamamoto, B. K., 2003. Effects of amphetamines on mitochondrial function: role of free radicals and oxidative stress. Pharmacology \& Therapeutics. 99, 45-53.

Callaghan, R.C., Cunningham, J.K., Sykes, J., Kish, S.J., 2012. Increased risk of Parkinson's disease in individuals hospitalized with conditions related to the use of methamphetamine or other amphetamine-type drugs. Drug Alcohol Depend. 120(1-3), 35-40.

Castillo, C., Morales, L., Alguacil, L.F., Salas, E., Garrido, E., Alonso, E., Pérez-García, C., 2009. Proteomic analysis of the nucleus accumbens of rats with different vulnerability to cocaine addiction. Neuropharmacology. 57(1), 41-48.

Clarke, P.R., Zhang, C., 2008. Spatial and temporal coordination of mitosis by Ran GTPase. Nat Rev Mol Cell Biol. 9, 464-477.

Cook, A.G., Conti, E., 2010. Nuclear export complexes in the frame. Curr Op Struct Biol. 20, $247-252$.

Del Olmo, N., Gramage, E., Alguacil, L.F., Pérez-Pinera, P., Deuel, T.F., Herradón, G., 2009. Pleiotrophin inhibits hippocampal long-term potentiation: a role of pleiotrophin in learning and memory. Growth Factors 27, 189-194.

Ezquerra, L., Herradon, G., Nguyen, T., Silos-Santiago, I., Deuel, T.F., 2005. Midkine, a newly discovered regulator of the renin-angiotensin pathway in mouse aorta: significance of the pleiotrophin/midkine developmental gene family in angiotensin II signaling. Biochem. Biophys. Res. Commun. 333, 636-643.

Ezquerra, L., Herradon, G., Nguyen, T., Silos-Santiago, I., Deuel, T.F., 2006. Midkine is a potent regulator of the catecholamine biosynthesis pathway in mouse aorta. Life Sci. 79, 10491055 .

Görg, A., Weiss, W., Dunn, M.J., 2004. Current two-dimensional electrophoresis technology for proteomics. Proteomics. 4(12), 3665-3685.

Gramage, E., Herradon, G., 2011. Connecting Parkinson's disease and drug addiction: common players reveal unexpected disease connections and novel therapeutic approaches. Curr. Pharm. Des. 17, 449-461. 
Gramage, E., Martin, Y. B., Ramanah, P., Perez-Garcia, C., Herradon, G., 2011. Midkine regulates amphetamine-induced astrocytosis in striatum but has not effects on amphetamine-induced striatal dopaminergic denervation and addcitive effects: Functional differences between pleiotrohin and midkine. Neuroscience. 190, 307-317.

Gramage, E., Putelli, A., Polanco, M.J., Gonzalez-Martin, C., Ezquerra, L., Alguacil, L.F., PerezPinera, P., Deuel, T.F., Herradon, G., 2010b. The neurotrophic factor pleiotrophin modulates amphetamine-seeking behaviour and amphetamine-induced neurotoxic effects: evidence from pleiotrophin knockout mice. Addict. Biol. 15, 403-412.

Gramage, E., Rossi, L., Granado, N., Moratalla, R., Herradon, G., 2010a. Genetic inactivation of Pleiotrophin triggers amphetamine-induced cell loss in the substantia nigra and enhances amphetamine neurotoxicity in the striatum. Neuroscience, 170, 308-316.

Granado, N., Ares-Santos, S., Oliva, I., O'Shea, E., Martin, E.D., Colado, M.I., Moratalla, R., 2011. Dopamine D2-receptor knockout mice are protected against dopaminergic neurotoxicity induced by methamphetamine or MDMA. Neurobiol. Dis. 42(3), 391-403.

Granado, N., Ares-Santos, S., O'Shea, E., Vicario-Abejon, C., Colado, M.I., Moratalla, R., 2010. Selective vulnerability in striosomes and in the nigrostriatal dopaminergic pathway after methamphetamine administration: early loss of TH in striosomes after methamphetamine. Neurotox. Res. 18, 48-58.

Granado, N., Escobedo, I., O'Shea, E., Colado, I., Moratalla, R., 2008a. Early loss of dopaminergic terminals in striosomes after MDMA administration to mice. Synapse. 62, 80-84.

Granado, N., O'Shea, E., Bove, J., Vila, M., Colado, M.I., Moratalla, R., 2008b. Persistent MDMA-induced dopaminergic neurotoxicity in the striatum and substantia nigra of mice. J. Neurochem. 107, 1102-1112.

Herradon. G., Ezquerra, L., 2009. Blocking receptor protein tyrosine phosphatase beta/zeta: a potential therapeutic strategy for Parkinson's disease. Curr. Med. Chem. 16, 3322-3329.

Herradon, G., Ezquerra, L., Gramage, E., Alguacil, L.F., 2009. Targeting the pleiotrophin/receptor protein tyrosine phosphatase beta/zeta signaling pathway to limit neurotoxicity induced by drug abuse. Mini Rev. Med. Chem. 9, 440-447.

Herradon, G., Ezquerra, L., Nguyen, T., Silos-Santiago, I., Deuel, T.F., 2005. Midkine regulates pleiotrophin organ-specific gene expression: evidence for transcriptional regulation and functional redundancy within the pleiotrophin/midkine developmental gene family. Biochem. Biophys. Res. Commun. 333, 714-721.

Hornbeck, P. V., Kornhauser, J.M., Tkachev, S., Zhang, B., Skrzypek, E., Murray, B., Latham, V., Sullivan, M., 2012. PhosphoSitePlus: a comprehensive resource for investigating the structure and function of experimentally determined post-translational modifications in man and mouse. Nucleic Acids Res. 40, D261-D270.

Huang, Q., Figueiredo-Pereira, M. E., 2010. Ubiquitin/proteasome pathway impairment in neurodegeneration: therapeutic implications. Apoptosis. 15, 1292-1311. 
Krasnova, I.N., Ladenheim, B., Cadet, J.L., 2005. Amphetamine induces apoptosis of medium spiny striatal projection neurons via the mitochondria-dependent pathway. Faseb. J. 19, 851-853.

Lee, W.H., Choi, J.S., Byun, M.R., Koo, K.T., Shin, S., Lee, S.K., Surh, Y.J., 2010. Functional inactivation of triosephosphate isomerase through phosphorylation during etoposideinduced apoptosis in HeLa cells: potential role of Cdk2. Toxicology. 278(2), 224-228.

Lessner, G., Schmitt, O., Haas, S.J., Mikkat, S., Kreutzer, M., Wree, A., Glocker, M.O., 2010. Differential proteome of the striatum from hemiparkinsonian rats displays vivid structural remodeling processes. J. Proteome. Res. 9(9), 4671-4687.

Li, M.D., Wang, J., 2007. Neuroproteomics and its applications in research on nicotine and other drugs of abuse. Proteomics Clin. Appl. 1, 1406-1427

Li, X. F., Wang, H. J., Qiu, P. M., Luo, H., 2008. Proteomic profiling of proteins associated with methamphetamine-induced neurotoxicity in different regions of rat brain. Neurochemistry International. 52, 256-264.

Li, Q., Zhang, R., Ge, Y.L., Mei, Y.W., Guo, Y.L., 2009. Effects of neuregulin on expression of MMP-9 and NSE in brain of ischemia/reperfusion rat. J. Mol. Neurosci. 38(2), 207-215.

Liemann, S., Lewit-Bentley, A.,1995. Annexins: a novel family of calcium- and membranebinding proteins in search of a function. Structure. 3, 233-237

Lovatt, M., Filby, A., Parravicini, V., Werlen, G., Palmer, E., Zamoyska, R., 2006. Lck regulates the threshold of activation in primary $\mathrm{T}$ cells, while both Lck and Fyn contribute to the magnitude of the extracellular signal-related kinase response. Mol. Cell. Biol. 26, 86558665 .

MacLeod, J. C., Sayer, R. J., Lucocq, J. M., Hubbard, M. J., 2004. ERp29, a general endoplasmic reticulum marker, is highly expressed throughout the brain. J. Comp. Neurol. 477, 29-42.

Mann, M., 2008. Can Proteomics Retire the Western Blot? J. Proteome Res., 7 (8), 3065.

Meng, K., Rodriguez-Pena, A., Dimitrov, T., Chen, W., Yamin, M., Noda, M., Deuel, T.F., 2000. Pleiotrophin signals increased tyrosine phosphorylation of beta beta-catenin through inactivation of the intrinsic catalytic activity of the receptor-type protein tyrosine phosphatase beta/zeta. Proc. Natl. Acad. Sci. USA. 97, 2603-2608.

Nakamura, E., Kadomatsu, K., Yuasa, S., Muramatsu, H., Mamiya, T., Nabeshima, T., Fan, Q.W., Ishiguro, K., Igakura, T., Matsubara, S., Kaname, T., Horiba, M., Saito, H., Muramatsu, T., 1998. Disruption of the midkine gene (Mdk) resulted in altered expression of a calcium binding protein in the hippocampus of infant mice and their abnormal behaviour. Genes Cells. 3, 811-822.

Narayanan, N.K., Narayanan, B.A., Nixon, D.W., 2004. Resveratrol-induced cell growth inhibition and apoptosis is associated with modulation of phosphoglycerate mutase B in human prostate cancer cells: two-dimensional sodium dodecyl sulfate-polyacrylamide gel electrophoresis and mass spectrometry evaluation. Cancer. Detect. Prev. 28(6), 443-452. 
Nersesova, L. S., 2011. Role of creatine kinase and its substrates in the central nervous system in norm and in various pathologies. Journal of Evolutionary Biochemistry and Physiology. $47,140-150$.

Nilsson, C.L., Dillon, R., Devakumar, A., Shi, S.D., Greig, M., Rogers, J.C., Krastins, B., Rosenblatt, M., Kilmer, G., Major, M., Kaboord, B.J., Sarracino, D., Rezai, T., Prakash, A., Lopez, M., Ji, Y., Priebe, W., Lang, F.F., Colman, H., Conrad, C.A., 2010. Quantitative phosphoproteomic analysis of the STAT3/IL-6/HIF1alpha signaling network: an initial study in GSC11 glioblastoma stem cells. J. Proteome. Res. 9, 430-443.

Obermajer, N., Doljak, B., Jamnik, P., Fonovic, U. P., Kos, J., 2009. Cathepsin X cleaves the Cterminal dipeptide of alpha- and gamma-enolase and impairs survival and neuritogenesis of neuronal cells. Int. J. Biochem. Cell. Biol. 41, 1685-1696.

Pariser, H., Ezquerra, L., Herradon, G., Perez-Pinera, P., Deuel, T.F., 2005a. Fyn is a downstream target of the pleiotrophin/receptor protein tyrosine phosphatase beta/zeta-signaling pathway: regulation of tyrosine phosphorylation of Fyn by pleiotrophin. Biochem. Biophys. Res. Commun. 332, 664-669.

Pariser, H., Herradon, G., Ezquerra, L., Perez-Pinera, P., Deuel, T.F., 2005b. Pleiotrophin regulates serine phosphorylation and the cellular distribution of beta-adducin through activation of protein kinase C. Proc. Natl. Acad. Sci. USA. 102, 12407-12412.

Prediger, R.D., Rojas-Mayorquin, A.E., Aguiar, A.S. Jr., Chevarin, C., Mongeau, R., Hamon, M., Lanfumey, L., Del Bel, E., Muramatsu, H., Courty, J., Raisman-Vozari, R., 2011. Mice with genetic deletion of the heparin-binding growth factor midkine exhibit early preclinical features of Parkinson's disease. J. Neural. Transm. 118(8), 1215-1225.

$\mathrm{Pu}$, C., Vorhees, C.V., 1995. Protective effects of MK-801 on methamphetamine-induced depletion of dopaminergic and serotonergic terminals and striatal astrocytic response: an immunohistochemical study. Synapse. 19, 97-104.

Sakaguchi, N., Muramatsu, H., Ichihara-Tanaka, K., Maeda, N., Noda, M., Yamamoto, T., Michikawa, M., Ikematsu, S., Sakuma, S., Muramatsu, T., 2003. Receptor-type protein tyrosine phosphatase zeta as a component of the signaling receptor complex for midkinedependent survival of embryonic neurons.

Scott, C.R., 2006. The genetic tyrosinemias. Am. J. Med. Genet. C. Semin. Med. Genet. 142, 121-126.

Shevchenko, A., Wilm, M., Vorm, O., Mann, M., 1996. Mass spectrometric sequencing of proteins from silver-stained polyacrylamide gels. Anal. Chem. 68, 850-858.

Sorzano, C.O., Arganda-Carreras, I., Thévenaz, P., Beloso, A., Morales, G., Valdés, I., PérezGarcía, C., Castillo, C., Garrido, E., Unser, M., 2008. Elastic image registration of 2-D gels for differential and repeatability studies and repeatability studies. Proteomics. 8(1), 62-65.

Spiess, C., Meyer, A.S., Reissmann, S., Frydman, J., 2004. Mechanism of the eukaryotic chaperonin: protein folding in the chamber of secrets. Trends. Cell. Biol. 14(11), 598604. 
Talvas, J., Obled, A., Sayd, T., Chambon, C., Mordier, S., Fafournoux, P., 2008. Phosphoproteomic approach to identify new targets of leucine deprivation in muscle cells. Anal. Biochem. 381(1), 148-150.

Tang, B., Seredenina, T., Coppola, G., Kuhn, A., Geschwind, D.H., Luthi-Carter, R., Thomas, E.A., 2011. Gene expression profiling of R6/2 transgenic mice with different CAG repeat lengths reveals genes associated with disease onset and progression in Huntington's disease. Neurobiol. Dis. 42(3), 459-467.

Taravini, I.R.E., Chertoff, M., Cafferata, E.G., Courty, J., Murer, M.G., Pitossi, F.J., Gershanik, O.S., 2011. Pleiotrophin over-expression provides trophic support to dopaminergic neurons in parkinsonian rats. Mol. Neurodegener. 6, 40

Westerlund, M., Galter, D., Carmine, A., Olson, L., 2005. Tissue- and species-specific expression patterns of class I, III, and IV Adh and Aldh 1 mRNAs in rodent embryos. Cell. Tissue. Res. 322(2), 227-236.

Wey, M.C., Fernandez, E., Martinez, P.A., Sullivan, P., Goldstein, D.S., Strong, R., 2012. Neurodegeneration and motor dysfunction in mice lacking cytosolic and mitochondrial aldehyde dehydrogenases: implications for Parkinson's disease. PLoS One. 7(2), e31522.

Xie, T., Tong, L., Barrett, T., Yuan, J., Hatzidimitriou, G., McCann, U.D., Becker, K.G., Donovan, D.M., Ricaurte, G.A., 2002. Changes in gene expression linked to methamphetamine-induced dopaminergic neurotoxicity. J. Neurosci. 22(1), 274-283.

Yamamoto, B.K., Bankson, M.G., 2005. Amphetamine neurotoxicity: cause and consequence of oxidative stress. Crit. Rev. Neurobiol. 17, 87-117.

Zhan, X., Desiderio, D.M., 2003a. A reference map of a human pituitary adenoma proteome. Proteomics 3, 699-713.

Zhan, X., Desiderio, D.M., 2003b. Differences in the spatial and quantitative reproducibility between two second-dimensional gel electrophoresis systems. Electrophoresis 24, 18341846. 


\section{FIGURE LEGENDS}

Figure 1. Representative silver stained 2D gel of the phosphoproteome from the

mouse striatum. Spots labelled with numbers showed significant differences in normalized optical density after comparing the 6 groups of animals and were identified by mass spectrometry.

Figure 2. Levels of phosphorylation in tyrosine of gamma-enolase (ENO2) in the striatum of PTN-/-, MK-/- and WT mice after amphetamine administration. The levels of phosphorylation of Tyr in saline (Sal)- and amphetamine (Amph)-treated PTN-/, MK-/- and WT mice ( $\mathrm{n}=4$ /group) were determined in Western blots probed with antiphospho-Tyr antibodies. Total ENO2 and Actin amounts were determined using antiENO2 and anti-actin antibodies. Upper graph: Ratio P-Tyr/ENO2 of optical density (OD) measurements corresponding to the phosphorylated form and total ENO2 protein levels respectively. Lower graph: Ratio ENO2/Actin of optical density (OD) measurements corresponding to the total ENO2 and actin protein levels respectively. Data show mean \pm S.E.M of the four individual samples from every experimental group.

Figure 3. Levels of phosphorylation in tyrosine of endoplasmic reticulum resident protein 29 (ERP29) in the striatum of PTN-/-, MK-/- and WT mice after amphetamine administration. The levels of phosphorylation of Tyr in saline (Sal)- and amphetamine (Amph)-treated PTN-/-, MK-/- and WT mice ( $\mathrm{n}=4$ /group) were determined in Western blots probed with anti-phospho-Tyr antibodies. Total ERP29 and Actin amounts were determined using anti-ERP29 and anti-actin antibodies. Upper graph: Ratio P-Tyr/ERP29 of optical density (OD) measurements corresponding to the phosphorylated form and total ERP29 protein levels respectively. Lower graph: Ratio ERP29/Actin of optical density (OD) measurements corresponding to the total ERP29 and actin protein levels respectively. Data show mean \pm S.E.M of the four individual samples from every experimental group. $* \mathrm{P}<0.05 \mathrm{vs}$. WT. 